\title{
The effects of microwave radiation generated by mobile phones on the tear film
}

\author{
Aurora Gajta ${ }^{1}$, Alexandra Maria Jurca ${ }^{2 *}$, Adriana Stănilă², Dan Stănilă \\ 1. Dept.of Ophthalmology, General Hospital Vrsac, Abraseviceva bb., Vrsac, Serbia \\ 2. Ophthalmology, University "Lucian Blaga” of Sibiu, Romania
}

\begin{abstract}
Electromagnetic radiation (ER) emitted by mobile phones and other modern devices has potentially harmful effects on ocular tissue. Their effects on the eye surface and tear film are little known so far. The aim of this paper was to investigate the effects of ER emitted by the phone mobile on the tear film. For this study, we selected a total of 50 subjects, young, healthy, without chronic treatment, who are not contact lens wearers and who have no history of ophthalmic surgery. Schirmer I test, tear pH and tear ferning test (TFT) were performed on all subjects before ("-pre") being exposed to ER emitted by the mobile phone and after ("-post") exposure for 5 minutes, the pH and TFT of the tears were performed. Following the analysis of the obtained results, we found that there are significant changes in tear quality and increased tear $\mathrm{pH}$, which over time can lead to tear film instability, damage to the eye surface and the appearance of dry eye syndrome.
\end{abstract}

Keywords: electromagnetic radiation, ocular surface, dry eye disease, tear filmt

Received: 10 $0^{\text {th }}$ March 2021; Accepted: $4^{\text {th }}$ June 2021; Published: $29^{\text {th }}$ June 2021

\section{Introduction}

Electromagnetic radiation (ER) emitted by mobile phones and other modern devices has potentially harmful effects on ocular tissue. Their effects on the eye surface and tear film are little known so far. Most mobile phones have a small antenna attached to or built into the phone. Because this antenna is very close to the user's head, there is a much higher radio frequency exposure than other types of radio frequency systems (1). Changes in the human body occur as a result of exposure to high levels of radio frequency energy. This energy leads to the production of large amounts of heat that the body is not able to eliminate (2). Given the significant potential impact of ER on public health, more and more research is being done in these areas nowadays.

Wang KJ et al. investigated the effect of microwave radiation on proliferative activity and on the cell cycle of crystalline epithelial cells in rabbits as well as the tolerance limit of exposure to microwave radiation. The results showed that

\footnotetext{
* Corresponding author: Alexandra Maria Jurca, Ophthalmology, University "Lucian Blaga" of Sibiu, Romania. E-mail: dr.alexandra.jurca@gmail.com
} 
after 8 hours of exposure to microwave radiation of $0.50,1.00$, and $2.00 \mathrm{~mW} / \mathrm{cm}^{2}$, epithelial cell proliferation is reduced, disordered cell arrangement occurs, cell contraction, detachment and inhibition of cellular DNA synthesis (3).

Environmental stress, including EM, has a negative impact on the lens and is considered a risk factor for the development of cataracts (4). More recent studies suggest that changes that occur during exposure to microwave radiation result from the absorption of electromagnetic energy. Protein folding during microwave radiation exposure comes from the resonant absorption of electromagnetic energy. This leads to a higher state of vibration of the protein or its aqueous coating and the occurrence of thermal imbalances. Another possibility is the absorption of high frequency microwaves by the aqueous layer. In conclusion, the changes that occur are reversible, but after prolonged exposure to microwave radiation, cumulative effects occur that can lead to cataracts $(5,6)$.

Novoselova et al (1999) demonstrated the stimulation of the immune potential of macrophages and $\mathrm{T}$ cells after five hours of exposure to $1 \mu \mathrm{W} /$ $\mathrm{cm}^{2}$ microwave radiation. Many recent studies demonstrate the effect of ER on ocular tissue, reactions to cellular stress after prolonged exposure can lead to increased inflammatory response and cellular apoptosis $(7,8)$. The effects of radiation on the tear film and the ocular surface are little known. Considering the composition of the tears, it is important to study the influence of radiation emitted by mobile phones on the tear film.

A healthy and stable tear film plays a vital role in maintaining both the optical quality of the eye and the integrity of the ocular surface. Any changes in the components of the tear film lead to symptoms of discomfort, visual disturbances, instability of the tear film, with potential damage of the ocular surface, accompanied by increased tear film osmolarity and inflammation of the ocular surface (9-11). In this paper we studied the effect of ER on the tear film by performing tear ferning test (TFT), as well as measuring tear $\mathrm{pH}$ on a number of 50 subjects, smokers and non-smokers, before exposure to electromagnetic radiation ("-pre") emitted by the mobile phone and after exposure to electromagnetic radiation ("-post") for five minutes. Schirmer I test was performed on all subjects before exposure, but not five minutes after exposure due to the duration of the test which could alter the results.

\section{Material and methods}

For this study, we selected a total of 50 subjects, young, healthy, without chronic treatment, who are not contact lens wearers and who have no history of ophthalmic surgery. The study design was approved by the ethics committee of General Hospital Vrsac, Serbia (no. 01-495/3, 01.04.2014). The study was conducted according to the criteria set by the declaration of Helsinki, we explained the non-invasive methods used for the research and each subject signed an informed consent before participating in the study.

For the Schirmer I test we used the standard test paper strip, inserted in the inferior conjunctival fornix, without anesthesia, after 5 minutes it was removed and the wet length of the strip was read, the results being noted in millimeters. For TFT, a tear was collected using a $0.5 \mu 1$ capillary tube, the contents were expelled on a degreased glass slide and allowed to dry at room temperature, after which the physical appearance of the tear was examined under a microscope. For the tear $\mathrm{pH}$, we used $\mathrm{pH}$ strips of paper inserted into the conjunctival sac and left to soak with tears, then the color obtained was compared with the color of the test scale. It should be noted that the material was taken from the same eye, which was exposed to radiation emitted by the mobile phone. The age of the subjects was between 19 and 30 
years with a mean of $23.22 \pm 0.43$. Thirty-six of the subjects were women $(72 \%)$ and 14 were men $(28 \%)$. Of the total subjects, 26 were smokers $(52 \%)$ and 24 were non-smokers (48\%).

The evaluation of the normality of the stratum data distribution was performed using the Kolmogorov Smirnov test, based on the SPSS software application, the Explore: Plots procedure, Normality plots with tests. Parametric testing of differences between groups was performed using the $\mathrm{T}$ test based on the Independent Samples $\mathrm{T}$ Test procedure. In the case of data that do not follow the normal distribution, the Wilcoxon and Sign tests were used, using Two Related Samples Tests from SPSS. Two independent Samples Tests were also used for the independent groups, with the Mann Whitney U test, from SPSS. The nonparametric statistical data correlation was studied based on the values of the Spearman's rho correlation coefficient, using Bivariate Correlations, Spearman, in SPSS. Some graphical representations were also made using SPSS.

\section{Results and discussions}

Schirmer I test - The minimum value was $12 \mathrm{~mm} / 5 \mathrm{~min}$, the maximum value was $18 \mathrm{~mm} /$ $5 \mathrm{~min}$ with an average value of $15.34 / 5 \mathrm{~min} \pm$ 1.96. We considered values higher than $10 \mathrm{~mm} /$ 5 minutes to be normal values (normal amount of tears).

Schirmer I test results in smokers ranged from $12 \mathrm{~mm} / 5 \mathrm{~min}$ ( $10 \%$ of patients) to $16 \mathrm{~mm} / 5 \mathrm{~min}$ ( $4 \%$ of patients). In non-smokers, test values ranged from $15 \mathrm{~mm} / 5 \mathrm{~min}$ (minimum value to 2 subjects) to $18 \mathrm{~mm} / 5 \mathrm{~min}$ (maximum value to 10 subjects). Analyzing the values obtained in the Schirmer I test, it is obvious that we found lower values in smokers than in non-smokers, which indicates an increased risk of dry eye syndrome in smokers. (Figure 1)

\section{Correlation of Schirmer I test values with dif- ferent parameters}

In order to determine possible correlations between the series related to the Schirmer test and other value data, the values of the Spearman 'rho correlation coefficient were calculated, between the data series related to the Schirmer I test and the values pH "-pre", pH "-post”, TFT “-pre", TFT "-post", respectively the age distribution of the subjects. The correlation coefficients have at least average and higher absolute dimensions (from $r=-0.629$ for correlation with the age of the statistical, up to $\mathrm{r}=-0.812$ for correlating Schirmer values with "-post” TFT values).

In order to determine possible correlations between the series related to the Schirmer I test and other value data, the values of the Spearman 'rho correlation coefficient were calculated, between the data series related to the Schirmer test and the values pH "-pre”, pH "-post”, TFT “-pre”, TFT "-post”, respectively the age of the subjects. The correlation coefficients have at least average and higher absolute dimensions (from $r=-0.629$ for correlation with the age of the statistical population up to $r=-0.812$ for correlating Schirmer values with "-post" TFT values. Moreover, all the values of the correlation coefficients are ensured statistically, with Sig. 2-tailed indicated low values, lower than 0.001 . It should be noted, however, that all correlation coefficients have

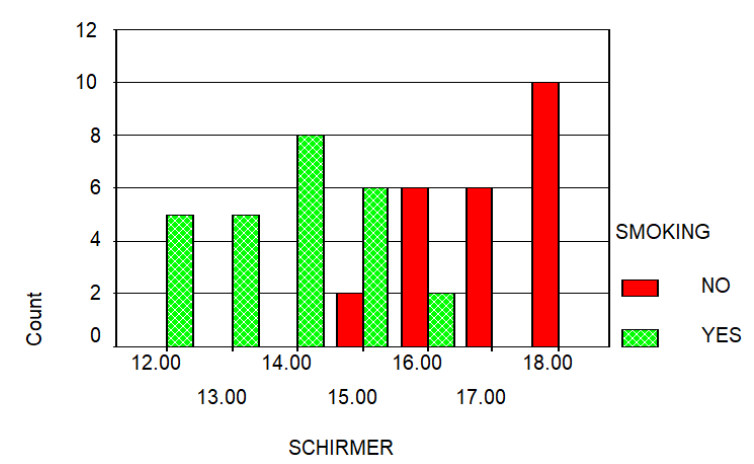

Fig. 1. Schirmer test values reported in smokers / non-smokers 
negative values, which indicates an inverse correlation. More precisely, the increase in the subjects' age implies a certain tendency of decreasing the Schirmer values $(\mathrm{r}=-0.629, \mathrm{p}<0.001)$. The upward trend in Schirmer values will also indicate some downward trend in "-pre" $\mathrm{pH}$ values $(\mathrm{r}=-0.748, \mathrm{p}<0.001), \mathrm{pH}$ “-post” $(\mathrm{r}=$ $-0.694, \mathrm{p}<0.001)$, TFT “-pre” $(\mathrm{r}=-0.766, \mathrm{p}<$ $0.001)$ and TFT "-post” ( $r=-0.812, \mathrm{p}<0.001)$. It should be noted that the decreasing trend of the measured values for both $\mathrm{pH}$ and TFT (when Schirmer values increase) is maintained without large variations in the correlation coefficient for both "-pre" and "-post" measurements.

\section{Testing the existence of possible differences between Schirmer's values depending on the sex of the subjects}

Statistical testing of the normal distribution of Schirmer I values leads to a value $\mathrm{p}=0.065$ when applying the Kolmogorov-Smirnov test. This indicates that the data come from a Gaussian distribution. In fact, medical practice confirms this result, usually patients undergoing the Schirmer I test often showed average values, while extreme values were found less often. Applying the $t$ test for independent data, it was observed that the male distribution did not differ significantly, regarding the average of the Schirmer I values $(\mathrm{mM}=15.07)$, from the female distribution $(\mathrm{mF}$ $=15.44$ ). Thus, the value of the test is $t=-0.710$ and Sig. 2-tailed is given by $p=0.482$, which indicates acceptance of the null hypothesis regarding the equality of the averages of the Schirmer I values, measured both in women as well as men.

\section{Testing differences between Schirmer I values in smokers/non-smokers}

Applying the $t$ test, for independent data, it was observed that the preponderance of smokers differed significantly, relative to the average of the Schirmer I values, from non-smokers. Specifically, the mean value for the smoker group was $\mathrm{mSMK}=13.80$, lower than the mean values Schirmer I mNO SMK=17.00 found for non-smokers. Thus, the value of the test is $\mathrm{t}=$ 9.920 and the Sig. 2-tailed is given by $p<0.001$ (assuming the equality of variances by Levene's Test with Sig. $=0.338, \mathrm{~F}=0.936$ ), which indicates the rejection of the null hypothesis regarding the equality of the averages of Schirmer I values measured in both smokers and non-smokers. (Figure 2)

Results presentation - TFT "-pre”: in 45 (90\%) subjects normal appearance was found in the crystallization test (type I and II) and type III was found in 5 subjects (5\%). Type IV was not found.

TFT "-post" emitted by the mobile phone for 5 minutes. No type I values were found, TFT type II was found in 19 samples (38\%), type III in 18 samples (36\%), and type IV in 13 samples $(26 \%)$.

In smokers, values found at TFT "-pre" were: type I was found in 3 smokers $(6 \%)$ and 18 non-smokers (36\%), type II was found in 18 smokers $(36 \%)$ and 6 non-smokers $(12 \%)$, and type III was found in 5 smokers $(10 \%)$ and 0 non-smokers $(0 \%)$.

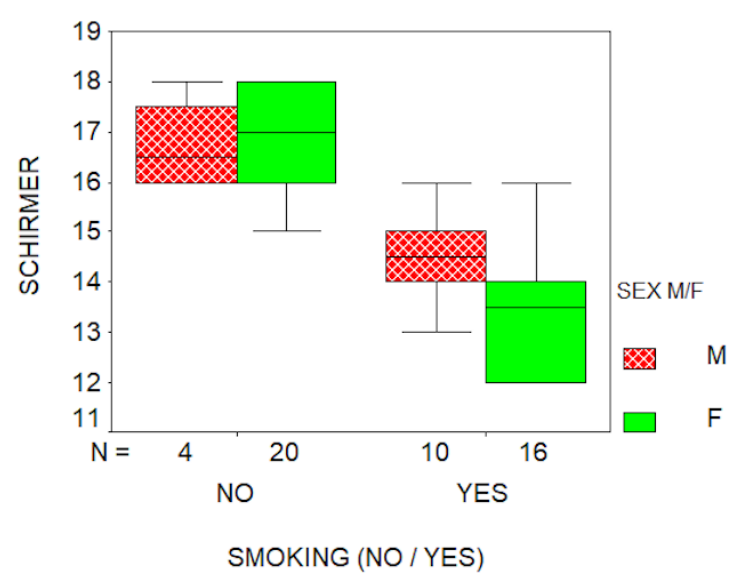

Fig. 2. Box-Plot Diagram - Schirmer I test values, grouped by smokers / non-smokers and by sex of subjects 
Smokers/ non-smokers TFT "-post” values, type I values were not found, type II was found in 19 samples (38\%), type III in 18 samples (36\%) and type IV in 13 samples (26\%). (Figure 3)

\section{TFT modification study}

Statistical tests were also performed using nonparametric tests. By applying the Kolmogorov-Smirnov test, a $\mathrm{p}$ value $<0.001$ is obtained for both the "-pre" and "-post" data group in the TFT calculation. This indicates a difference from the normal curve and can probably be explained, as in the previous situation, by the small number of descriptors of the TFT stage values (I, II, III, IV).

TFT status values were also analyzed both before and after the use of mobile phone. Thus, out of the 50 subjects, in 47 of them an increase in the TFT stage was found and in 3 of them a constant preservation of the stage was observed. No cases were observed in which the TFT stage decreased after using mobile phone.

These aspects can also be viewed graphically based on the Box-Plot diagram below, observing the positioning at the bottom of the TFT axis of the TFT values measured "-pre", compared with the TFT values measured "-post", where most are positioned higher. (Figure 4)

The differences between the TFT values measured "-pre" compared to the TFT values mea-

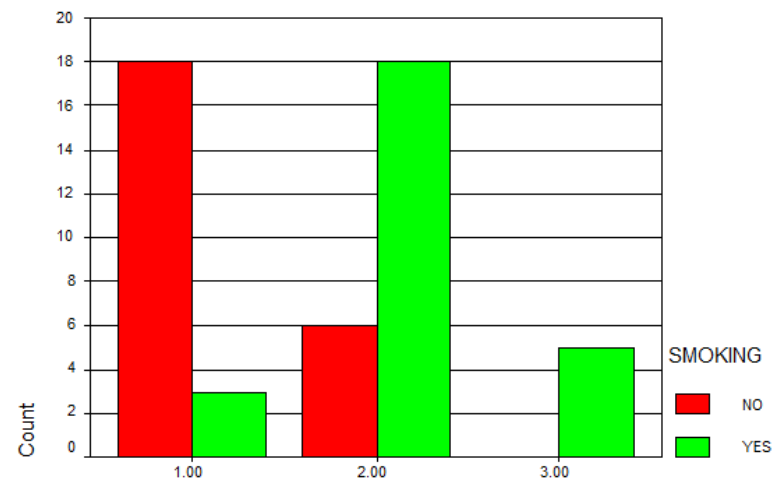

TFT_PRE sured "-post" indicated above, were statistically evaluated based on the nonparametric Wilcoxon Signed Ranks Test. The differences between the two data series are statistically significant, with $\mathrm{p}<0.001$. In addition, similar results were also indicated by the Sign Test, with high statistical significance, $\mathrm{p}<0.001$.

The issue was then to assess the possible differences between the group of smokers (1) and non-smokers (0), respectively, regarding the values of TFT stages, evaluated both before (" pre") and after ("-post") using the mobile phone. The Mann Whitney test was used to confirm these observations. It confirmed the existence of significant differences between the group of

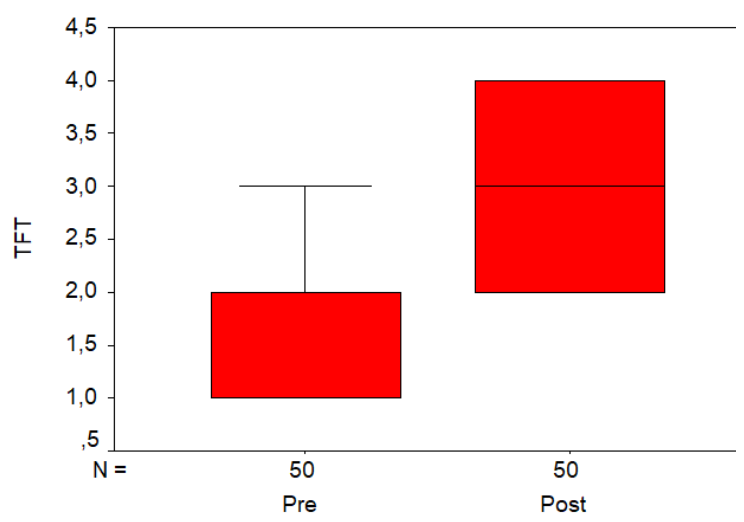

Fig. 4. Box-Plot Diagram - TFT stage values, measured "-pre" and "-post" mobile phone use

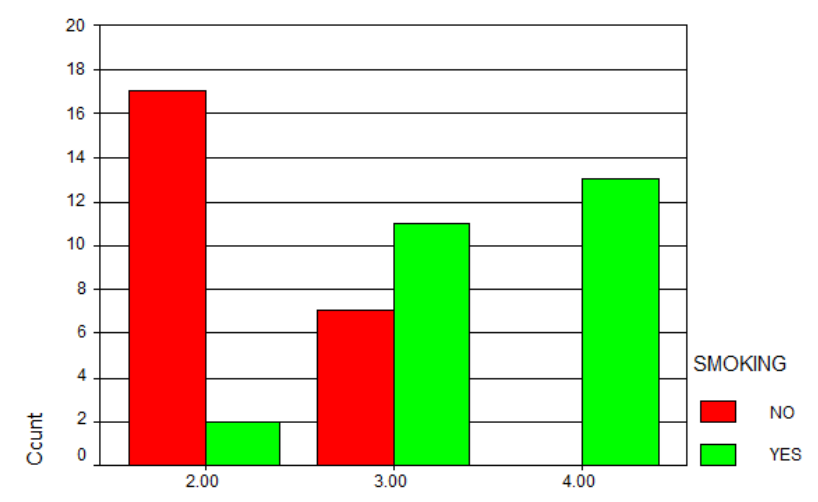

TFT_POST

Fig. 3. TFT "-pre" and TFT "-post" values in smokers and non-smokers 
smokers and non-smokers, for the TFT measurement made before ("-pre") and after ("-post") using the mobile phone. These details are revealed by the probability values $\mathrm{p}$ (As. Sig. 2-tailed), low for each case, with $\mathrm{p}<0.001$.

\section{Aspects of the TFT}

The classification was made after the Rolando classification (12) where type I has a lot of ferns with multiple trees, well-represented and present on the entire surface of the drop. Type II, abundance of ferns with free spaces between them, type III has a rare or even unique fern, with large free spaces, and type IV demonstrates the absence of ferns, only some mucus threads being visible. (Figure 5)

In the past, some authors considered that the appearance of fern leaf, the crystallized tear, is due to the amount of mucus present in the tear film (13). With the advent of new technologies,
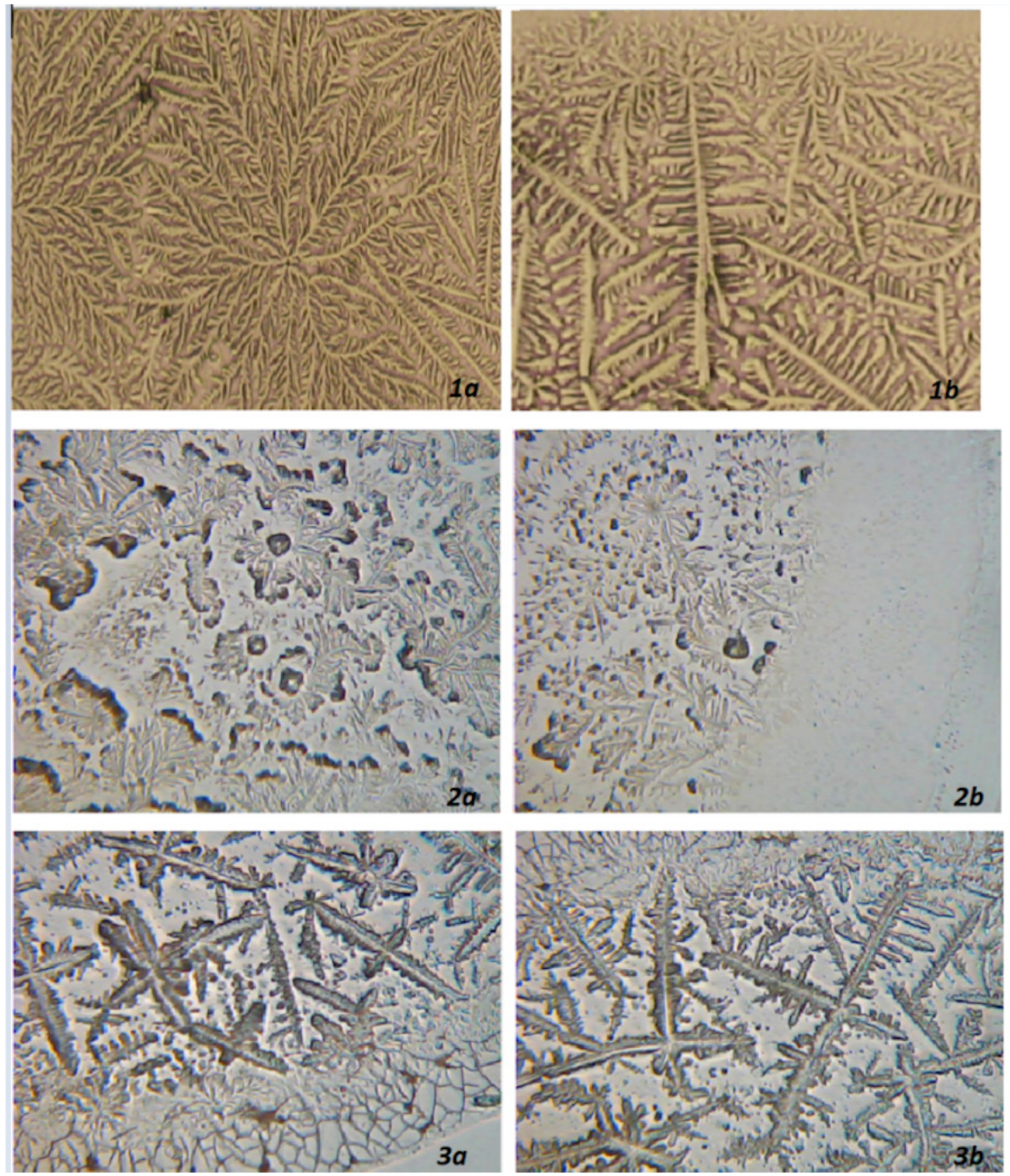

Fig. 5. TFT appearance before exposure to ER (1a, 1b), TFT appearance after exposure for 5 minutes to ER ( 2a, 2b), TFT appearance 10 min after cessation of ER exposure( 3a, 3b) 
biochemical analyses identify other factors that are part of the TFT process, such as the concentration of ions in tears (14). The crystallization of the tear in the form of "fern leaf", according to some authors, depends on the amount of protein in tears (12). The low amount of protein in tears, in people with dry eye syndrome, forms different patterns of fern leaf. The test correlates with clinical signs and is an objective guide in tracking the effects of dry eye therapy $(15,16)$. At 10 minutes after the exposure to ER emitted by the mobile phone, the physical appearance of the tear remains altered.

Study of change in $\mathbf{p H}$ - Statistical tests were performed using nonparametric tests due to observed differences from the normal distribution, noted by applying the Kolmogorov-Smirnov test $(\mathrm{p}<0.001)$, for the "-pre" and "-post" data group. The distance from the normal curve can be explained by the small number of descriptors of $\mathrm{pH}$ values (7, 8 and 9). The $\mathrm{pH}$ checks tested for the same group of individuals, before and after using the mobile phone show some value changes. Specifically, referring to the group of 50 subjects analyzed, in 40 of them increases in $\mathrm{pH}$ value were observed, and 10 of them have constant values. However, no decrease in $\mathrm{pH}$ values was observed after using the phone.

We considered the $\mathrm{pH}$ values marked with 7 as normal values (values between $7-7.5$ ), with 8 we noted the values between $7.5-8$, and with 9 we noted the values $>8$. Values greater than 8 are found in people with dry eye syndrome. These differences can be viewed graphically by following the Box-Plot diagram (Figure 6). The group of values framed by rectangles (values between the first and third quartiles), relating to the $\mathrm{pH}$ values measured "-pre" is placed at the bottom of the axis of the $\mathrm{pH}$ values, compared with similar data from "-post" testing that are positioned higher and mostly higher.

PH values "-pre": $>7$ were found in 31 subjects $(62 \%), \leq 8$ in 19 subjects (38\%), and between 8-9 in 0 subjects.
PH values "-post" for 5 minutes: $>7$ to 7 subjects $(14 \%), \leq 8$ to 17 subjects (34\%), and 8-9 to 26 subjects or $52 \%$.

Elevated $\mathrm{pH}$ values are found in rosacea and dry eye syndrome. Following exposure to ER emitted by the cell phone, the $\mathrm{pH}$ rises to $86 \%$ of the samples.

For the statistical verification of the above observations, the Wilcoxon Signed Ranks Test was used. The differences observed previously are confirmed, the value of the probability $\mathrm{p}$ being low, more precisely $\mathrm{p}<0.001$. This indicates a statistical significance of differences. In addition, Sign Test was applied. The results are similar, $\mathrm{p}<0.001$, which also provides statistically the differences observed previously, between the $\mathrm{pH}$ values measured on the same group of people, on the one hand before using the mobile phone and on the other hand after using it ("-pre" vs. "-post").

Following the Box-Plot diagram below, we notice that the group of smokers (1) often has values positioned higher on the scale of $\mathrm{pH}$ values, compared with the group of non-smokers (0), which have lower values. These observations are found in both "-pre" and "-post" testing. In order to confirm these differences, between the $\mathrm{pH}$ values for the smoking group and between the $\mathrm{pH}$

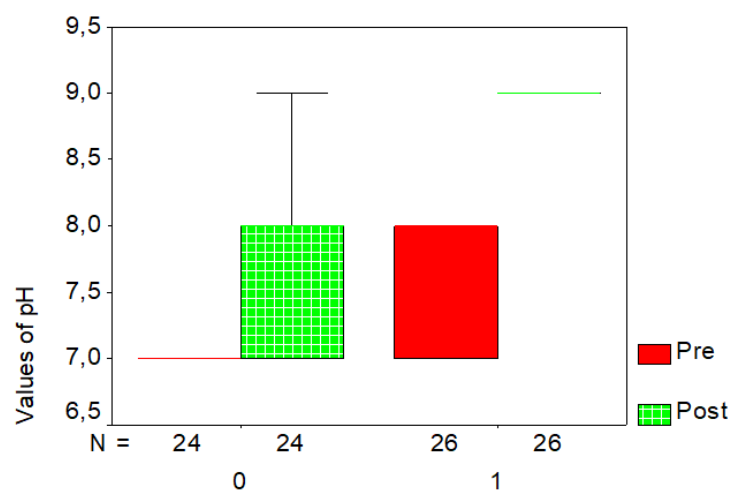

Fig. 6. Box-Plot diagram of $\mathrm{pH}$ values grouped by number of smokers and non-smokers (measured "-pre" and "-post") 
values measured in the non-smoking group, the Mann-Whitney test was used.

The differences are statistically significant, both in the case of measurements performed before using the mobile phone $(p<0.001)$ and in the case of $\mathrm{pH}$ measurements performed after using the phone $(\mathrm{p}<0.001)$. (Figure 6$)$

\section{Testing the differences between $\mathrm{pH}$ and TFT values, depending on the sex of the subjects}

In addition, we tested the possible existence of differences between $\mathrm{pH}$ and TFT values depending on the sex of the subjects. The evaluations were performed using the Mann-Whitney test, for all data series: $\mathrm{pH}$ "-pre", $\mathrm{pH}$ "-post", TFT "-pre", TFT "-post". The differences between the female and male groups are not noticeable, whether the measurements were made before or after exposure to the mobile phone. If, however, they appear, they are low and have no statistical significance $(p=0.662$ in the case of $\mathrm{pH}$ testing "-pre", $p=0.247$ in the case of $\mathrm{pH}$ testing "-post" mobile phone use, respectively $p=0.495$ in the case of TFT testing "-pre", and $p=0.448$ in the case of TFT testing "-post" mobile phone use).

\section{Conclusion}

The $\mathrm{pH}$ values measured before using the mobile phone are most often lower compared with similar data corresponding to the $\mathrm{pH}$ test after using the phone, the differences having a statistical significance.

The group of smokers usually has values positioned higher on the scale of $\mathrm{pH}$ values, compared with the group of non-smokers. The differences are statistically significant, both when the measurements were taken before and after using the mobile phone. The TFT stage values analyzed before using the mobile phone are usually lower than the TFT values measured after using the mobile phone and the differences are statistically significant.
The TFT values measured before using the phone but also those measured after using the phone, for non-smokers are often placed lower compared with those in the group of smokers and confirm the existence of statistically significant differences.

The values of the correlation coefficient, between the data series related to the Schirmer test values, the $\mathrm{pH}$ and TFT values, before and after the use of the mobile phone, or the age of the people, indicate at least average dimensions of correlations but also higher and are statistically assured. All correlation coefficients have negative values, so they indicate an inverse correlation. The decreasing trend of $\mathrm{pH}$ and TFT values as Schirmer values increase is maintained without large variations in the correlation coefficient regardless whether the measurements were made before or after the use of the mobile phone.

The average Schirmer values do not differ statistically in the male group compared with the female group. However, the average Schirmer value for smokers was lower compared with the average Schirmer value found in non-smokers, statistically significant.

Regardless whether the measurements were made before or after exposure to the mobile phone, there are no differences between the female and male groups in terms of $\mathrm{pH}$ or TFT values.

The ER emitted by mobile phones significantly changes the physical appearance of the tear, affecting the quality of the tear film.

The $\mathrm{pH}$ of tears, after exposure to ER increases. In smokers, test results show more pronounced changes in $\mathrm{pH}$ and physical appearance of the tear (TFT), which indicates an increased risk for dry eye syndrome.

The combination of several risk factors presents more pronounced changes in the tear film and an increased risk for dry eye syndrome. 


\author{
Abbreviations \\ ER - electromagnetic radiation; \\ "-pre" - before exposure to electromagnetic ra- \\ diation; \\ "-post" - after exposure to electromagnetic ra- \\ diation; \\ TFT - tear ferning test
}

\section{Authors' contribution}

G. A. (Patient data collection; Data analysis and statistics; Methodology; Investigation; Writing original draft)

J. A. (Patient data collection; Data analysis and statistics; Methodology; Investigation; Writing original draft; Writing -review \& editing)

S. A. (Patient data collection; Methodology; Supervision; Validation; Investigation)

S. D. (Patient data collection; Investigation; Methodology)

\section{Conflict of interest}

The authors declare no conflict of interest.

\section{References}

1. Vignal R, Crouzier D, Dabouis V, Debouzy JC. Effets des ondes hyperfréquences des téléphones mobiles et des radars sur l'oeil [Effects of mobile phones and radar radiofrequencies on the eye]. Pathol Biol (Paris). 2009 Sep;57(6):503-8. DOI: 10.1016/j.patbio.2008.09.003

2. George DF, Bilek MM, McKenzie DR. Non-thermal effects in the microwave induced unfolding of proteins observed by chaperone binding. Bioelectromagnetics. 2008 May;29(4):324-30. DOI: 10.1002/bem.20382

3. Wang KJ, Yao K, Lu DQ, Jiang H, Tan J, Xu W. [Effect of low-intensity microwave radiation on proliferation of cultured epithelial cells of rabbit lens]. Zhonghua Lao Dong Wei Sheng Zhi Ye Bing Za Zhi. 2003 Oct;21(5):346-9.

4. Lixia S, Yao K, Kaijun W, Deqiang L, Huajun H, Xiangwei G, Baohong W, Wei Z, Jianling L, Wei W. Effects of $1.8 \mathrm{GHz}$ radiofrequency field on DNA damage and expression of heat shock protein 70 in human lens ep- ithelial cells. Mutat Res. 2006 Dec 1;602(1-2):135-42. DOI: 10.1016/j.mrfmmm.2006.08.010

5. Wang J, Koyama S, Komatsubara Y, Suzuki Y, Taki M, Miyakoshi J. Effects of a $2450 \mathrm{MHz}$ high-frequency electromagnetic field with a wide range of SARs on the induction of heat-shock proteins in A172 cells. Bioelectromagnetics. 2006; 27:479-86. DOI: 10.1002/ bem.20226

6. Yu Y, Yao K. Non-thermal cellular effects of low power microwave radiation on the lens and lens epithelial cells. J Int Med Res. 2010;38(3):729-36. DOI: 10.1177/147323001003800301

7. Kivrak EG, Yurt KK, Kaplan AA, Alkan I, Altun G. Effects of electromagnetic fields exposure on the antioxidant defense system. J Microsc Ultrastruct. 2017;5(4):167-6. DOI: 10.1016/j.jmau.2017.07.003

8. Elder JA. Ocular effects of radiofrequency energy. Bioelectromagnetics. 2003;Suppl 6:S148-61. DOI: 10.1002/bem. 10117

9. Munshi S, Varghese A, Dhar-Munshi S. Computer vision syndrome-A common cause of unexplained visual symptoms in the modern era. Int J Clin Pract. 2017:71. DOI: $10.1111 /$ ijcp. 12962

10. Choi JH, Li Y, Kim SH, Jin R, Kim YH, Choi W, You IC, Yoon KC. The influences of smartphone use on the status of the tear film and ocular surface. PLoS One. 2018 Oct 31;13(10):e0206541. DOI: 10.1371/journal. pone. 0206541

11. Tavares Fde P, Fernandes RS, Bernardes TF, Bonfioli AA, Soares EJ. Dry eye disease. Semin Ophthalmol. 2010 May;25(3):84-93. DOI: 10.3109/08820538.2010.488568

12. Sharanjeet-Kaur, Ho CY, Mutalib HA, Ghazali AR. The Relationship Between Tear Ferning Patterns and Non-invasive Tear Break-up Time in Normal Asian Population. J Optom. 2016;9(3):175-81. DOI: 10.1016/j.optom.2015.10.004

13. Am M, Ra F, El-Naggar AH, Tm A; Akhtar S. Structure and microanalysis of tear film ferning of camel tears, human tears, and Refresh Plus. Mol Vis. 2018 Apr 16; 24:305-14.

14. Masmali AM, Purslow C, Murphy PJ. The tear ferning test: a simple clinical technique to evaluate the ocular tear film. Clin Exp Optom. 2014 Sep; 97(5):399-406. DOI: $10.1111 /$ cxo. 12160

15. Bârjovanu F, Mocanu C, Mercuţ G. Testul de cris- 
talizare a lacrimilor in sindromul sicca [The tear crystallization test in sicca syndrome]. Oftalmologia. 2006;50(4):54-9.
16. Norn M. Quantitative tear ferning. Clinical investigations. Acta Ophthalmol (Copenh). 1994 Jun;72(3):36972. DOI: $10.1111 / j .1755-3768.1994 . t b 02775 . x$ 